\title{
MicroRNA-327 regulates cardiac hypertrophy and fibrosis induced by pressure overload
}

\author{
YUE JI ${ }^{1,2^{*}}$, MING QIU ${ }^{1,2^{*}}$, YEJIAO SHEN ${ }^{1,2}$, LI GAO $^{1,2}$, YAQING WANG ${ }^{1,2}$, WEI SUN $^{1}$, \\ XINLI LI ${ }^{1}$, YAN LU ${ }^{1}$ and XIANGQING KONG ${ }^{1}$ \\ ${ }^{1}$ Department of Cardiology, The First Affiliated Hospital of Nanjing Medical University; \\ ${ }^{2}$ Department of Cardiology, Graduate Institution of Integrated Medicine, \\ Nanjing Medical University, Nanjing, Jiangsu 210029, P.R. China
}

Received August 11, 2017; Accepted January 19, 2018

DOI: $10.3892 /$ ijmm.2018.3428

\begin{abstract}
MicroRNA (miRNA/miR) dysregulation has been reported to be fundamental in the development and progression of cardiac hypertrophy and fibrosis. In the present study, miR-327 levels in fibroblasts were increased in response to cardiac hypertrophy induced by transverse aortic constriction with prominent cardiac fibrosis, particularly when compared with the levels in unstressed cardiomyocytes. In neonatal rat cardiac fibroblasts, induced expression of miR-327 upregulated fibrosis-associated gene expression and activated angiotensin II-induced differentiation into myofibroblasts, as assessed via $\alpha$-smooth muscle actin staining. By contrast, miR-327 knockdown mitigated angiotensin II-induced differentiation. Cardiac fibroblast proliferation was not affected under either condition. In a mouse model subjected to transverse aortic constriction, miR-327 knockdown through tail-vein injection reduced the development of cardiac fibrosis and ventricular dysfunction. miR-327 was demonstrated to target integrin $\beta 3$ and diminish the activation of cardiac fibroblasts. Thus, the present study supports the use of miR-327 as a therapeutic target in the reduction of cardiac fibrosis.
\end{abstract}

Correspondence to: Dr Yan Lu or Professor Xiangqing Kong, Department of Cardiology, The First Affiliated Hospital of Nanjing Medical University, 300 Guangzhou Road, Nanjing, Jiangsu 210029, P.R. China

E-mail: kitty1985517@126.com

E-mail: kongxq_njmu@outlook.com

Abbreviations: BW, body weight; EF, ejection fraction; FBS, fetal bovine serum; FGF, fibroblast growth factor; HW, heart weight; LV, left ventricle; LVPW, left ventricular posterior wall; MAPK, mitogen-activated protein kinase; NRCF, neonatal rat cardiac fibroblasts; TAC, transverse aortic constriction; TL, tibia length

*Contributed equally

Key words: microRNA-327, cardiac hypertrophy, fibrosis, integrin $\beta 3$

\section{Introduction}

Mechanical and chemical stimuli, including changes in pressure and changes in the levels of cytokines, hormones or growth factors, trigger cardiac hypertrophy, which subsequently leads to cardiomyopathy and heart failure $(1,2)$. Despite improvements in treatment strategies and the current understanding of the pathology, heart failure continues to be the leading cause of mortality worldwide. Pathological hypertrophy, as well as other types of cardiac injury, may trigger cardiac fibrosis, which in turn leads to adverse ventricular remodeling and heart failure (3-5). Therefore, identifying therapeutics that prevent the onset of pathological cardiac hypertrophy is of great interest.

Aberrant expression of microRNAs (miRNAs/miRs), small noncoding RNAs that regulate gene expression at the post-transcriptional level (6-8), serves a distinct role in disease pathology by disrupting signaling cascades and gene-regulatory networks. For instance, miRNA has been linked to adverse cardiac remodeling and fibrosis (4,9-11). The current understanding of the exact role of miRNA in cardiac hypertrophy has been increased by studies using mouse models coupled with gain- and loss-of-function strategies $(12,13)$.

In a previous study, through the use of miRNA arrays, it was demonstrated that miR-327 levels were enhanced in mouse left ventricle (LV) tissues following myocardial infarction (MI), suggesting that miR-327 may be involved in cardiac hypertrophy and fibrosis induced by pressure overload (3). In the present study, it was demonstrated that miR-327 expression was increased in response to transverse aortic constriction (TAC) in the LV of mice. Angiotensin II-induced differentiation of cardiac fibroblasts into myofibroblasts was increased by miR-327 overexpression. By contrast, miR-327 knockdown inhibited angiotensin II-induced differentiation. In the mouse model, downregulation of miR-327 using antagomiR prevented pressure overload-induced hypertrophy and fibrosis. In addition, integrin $\beta 3$ (ITGB3) was identified as a target gene of miR-327. The present study suggests that cardiac fibrosis is promoted by miR-327; therefore, therapeutics aimed towards inhibiting miR-327 may be effective treatments for cardiac fibrosis. 


\section{Materials and methods}

Antibodies and reagents. Antibodies targeting GAPDH (cat. no. 5174), phosphorylated extracellular signalregulated protein kinases $1 / 2$ (p-ERK1/2, cat. no. 4370), p-p38 (cat. no. 4511), p-c-Jun N-terminal protein kinase (p-JNK, cat. no. 9251), matrix metalloproteinase-9 (MMP-9, cat. no. 13667) and proliferating cell nuclear antigen (PCNA, cat. no. 13110) were purchased from Cell Signaling Technology, Inc. (Danvers, MA, USA). Collagen type I $\alpha 1$ (Col1a1, cat. no. ab34710) and $\alpha$-smooth muscle actin ( $\alpha$-SMA, cat. no. ab32575) were purchased from Abcam (Cambridge, MA, USA). Horseradish peroxidase (HRP)-conjugated secondary antibodies targeting rabbit and mouse were from Cell Signaling Technology, Inc. Alexa Fluor 488-conjugated streptavidin antibody (cat. no. 111-545-003) was from Jackson ImmunoResearch (West Grove, PA, USA) and angiotensin II was obtained from Sigma-Aldrich (Merck KGaA, Darmstadt, Germany). miR-327 agomiR (cat. no. miR4000561), antagomiR (cat. no. miR30000561) and scrambled controls (for control of agomiR, cat. no. miR4000561; for control of antagomiR, cat. no. miR4000561 were purchased from Guangzhou RiboBio Co., Ltd. (Guangzhou, China).

Ethics statement. The Institutional Animal Care and Use Committee of Nanjing Medical University (Nanjing, China) approved all animal protocols. As described below, a cardiac hypertrophy and heart failure model was developed using TAC surgery (14). All procedures involving animals were in accordance with the Guide for the Care and Use of Laboratory Animals published by the National Institutes of Health (no. 85-23; revised 1996) and the study protocol was approved by The Institutional Animal Care and Use Committee (IACUC) of Nanjing Medical University (Nanjing, China; nos. IACUC1701020 and IACUC-14030149). The mice were used for TAC surgery, while the rats were subjected to isolation of cardiac fibroblasts for in vitro experiments.

Animals and TAC surgery. A total of 72 male C57BL/6 mice (age, 8 weeks; weight, 22-24 g) obtained from the model animal research center of Nanjing University (Nanjing, China) were stratified into groups of 6 animals each: Sham NC antagomiR, TAC NC antagomiR, Sham miR-327 antagomiR and TAC miR-327 antagomiR. Mice were maintained under appropriate barrier conditions under a 12-h light/dark cycle, constant temperature range from $20-22^{\circ} \mathrm{C}$, humidity range from $50-60 \%$, and received food and water ad libitum. TAC is the primary method for inducing cardiac hypertrophy in mice and rats (15). In brief, mice were deeply anesthetized with $2 \%$ isoflurane, maintained under $1.5-2.0 \%$ isoflurane and intubated using a volume-cycled ventilator, and a midline incision was made above the sternum. The muscles were carefully separated until the trachea was visualized. A chest retractor was used to retract the sternum, and a partial left-side thoracotomy was performed to the second rib using blunt-ended, spring-action scissors. The lobes of the thymus were separated and the aortic arch was cleaned of fat tissue using blunttipped, $45^{\circ}$-angled forceps. A sterile saline-soaked 7-0 silk suture was placed between the innominate and left common carotid arteries using $90^{\circ}$-curved forceps. A 27 -gauge needle fragment was aligned parallel to the transverse aorta, and the suture was loosely tightened around the transverse aorta twice. The two knots were tied against the needle in rapid succession. Following removal of the needle, a $0.4-\mathrm{mm}$ diameter constriction remained. The sham surgery mice were subjected to the same procedure except for the tightening around the transverse aorta.

Animal treatment. To determine whether miR-327 inhibition prevents cardiac fibrosis in vivo, antagomiR, a 2'-O-methyl5'cholesterol-modified miR-327 inhibitor, or a scrambled control (aforementioned; Guangzhou RiboBio Co., Ltd.) was administered to the mice at $80 \mathrm{mg} / \mathrm{kg}$ body weight via tail-vein injection, daily for 3 days prior to TAC surgery ( $n=6$ per group).

Cardiac imaging. Transthoracic, two-dimensional M-mode echocardiography was performed using a Vevo 770 high-resolution in vivo imaging system with a 30-MHz transducer (VisualSonics, Inc., Toronto, ON, Canada). Echocardiographic studies were performed at $28 \mathrm{~h}$ following TAC surgery. The LV mass, LV wall thickness, ejection fraction (EF) and percent of fractional shortening were calculated as previously described (16).

Isolation of neonatal rat cardiomyocytes and fibroblasts. Sprague Dawley rats (age, 1-3 days; weight, 5-6 g) obtained from Beijing Vital River Laboratory Animal Technology Co., Ltd. (Beijing, China) were used to isolate cardiac fibroblasts. The ventricular tissue was finely minced and digested in buffer $\left(37^{\circ} \mathrm{C}\right.$, with agitation, $\left.30 \mathrm{~min}\right)$ containing trypsin (6 mg/ml, Sigma-Aldrich; Merck KGaA) and collagenase type II $(4 \mathrm{mg} / \mathrm{ml}$, Worthington Biochemical Corporation, Lakewood, NJ, USA) at a ratio of 3:2. The resultant cell suspensions were pelleted by centrifugation (room temperature, $120 \mathrm{~g}$, 3 min), resuspended in Dulbecco's modified Eagle's medium (Gibco; Thermo Fisher Scientific, Inc., Waltham, MA, USA) with $100 \mathrm{U} / \mathrm{ml}$ penicillin, $100 \mu \mathrm{g} / \mathrm{ml}$ streptomycin and $10 \%$ fetal bovine serum (FBS; Sciencell Research Laboratories, Inc., San Diego, CA, USA), 5\% horse serum (Hyclone; GE Healthcare, Little Chalfont, UK) and seeded into culture plates. Fibroblasts were allowed to attach for $2 \mathrm{~h}$ at $37^{\circ} \mathrm{C}$ and $5 \% \mathrm{CO}_{2}$. The unattached cardiomyocytes were then plated in culture plates coated with $10 \mathrm{mg} / \mathrm{ml}$ gelatin (Sigma-Aldrich; Merck KGaA).

Cell transfection. Cardiac fibroblast experiments were performed in low-serum (1\% FBS) medium. At passage 3, cardiac fibroblasts $\left(5 \times 10^{4} / \mathrm{ml}\right)$ were transfected with miRNA agomiR $(100 \mathrm{nM})$, antagomiR $(200 \mathrm{nM})$ or the scrambled controls (100 or $200 \mathrm{nM}$ ) for $48 \mathrm{~h}$ at $37^{\circ} \mathrm{C}$. Subsequently, cells were treated with $1 \mu \mathrm{M}$ angiotensin II (Sigma-Aldrich; Merck KGaA) for $24 \mathrm{~h}$ at $37^{\circ} \mathrm{C}$.

Immunohistochemistry. At 4 weeks following TAC surgery, mice were deeply anesthetized with $2 \%$ isoflurane, maintained at $1.5-2 \%$ isoflurane and intubated using a volume-cycled ventilator. The mice were perfused with PBS, followed by $4 \%$ buffered paraformaldehyde. The hearts were isolated, subjected to PBS perfusion and formalin-fixed overnight. The head and apex of the heart were divided, tissues were embedded in paraffin blocks and tissue sections ( $n=20-25$ per 
heart; thickness, $4 \mu \mathrm{m}$ ) were prepared. To assess fibrosis, the sections were stained with Masson's Trichrome and picrosirius red according to standard procedures $(16,17)$. Collagen and non-collagen components were red- and orange-stained, respectively. The percentage of the tissue stained was determined (in 5 fields per sample) and statistically significant differences between groups were determined.

Neonatal rat cardiac fibroblasts (NRCF) were fixed in $4 \%$ paraformaldehyde and permeabilized with $0.2 \%$ Triton X-100 in PBS for $10 \mathrm{~min}$. NRCF were blocked with $5 \%$ normal serum that matched the species used to generate the secondary antibody and incubated with $\alpha$-SMA primary antibody (1:500 dilution) overnight at $4{ }^{\circ} \mathrm{C}$, followed by fluorochrome-conjugated secondary antibodies (1:500 dilution) for $1 \mathrm{~h}$ at room temperature. DAPI was used to counterstain nuclei. Images were captured with a Carl Zeiss Axioskop microscope (Carl Zeiss AG, Oberkochen, Germany).

Cell proliferation assay. Cell proliferation was measured in real-time using the xCELLigence system (Roche Applied Science, Penzberg, Germany) (18). This system measures electrical impendence caused by adherent cells to evaluate proliferation in real time (19). Cells were seeded at 2,000 cells per well and allowed to attach for $12 \mathrm{~h}$. The cell number, and therefore cell proliferation, was correlated with the relative change in impedance when measurements were made with and without cells through a unit-less parameter referred to as the Cell Index (20). The Cell Index at each time-point $(0,1$, $6,12,18,24,30,36,42,48,54,60$ and 66 h) was normalized to the value recorded at time-point 0/baseline, which was immediately following transfection with miRNA agomiR (100 $\mathrm{nM})$, antagomiR $(200 \mathrm{nM})$ or the scrambled controls (100 or $200 \mathrm{nM}$ ) for $24 \mathrm{~h}$ at $37^{\circ} \mathrm{C}$, and treatment with $1 \mu \mathrm{M}$ angiotensin II for $24 \mathrm{~h}$ at $37^{\circ} \mathrm{C}$.

Western blot analysis. The phosphorylated and total protein content from cultured cells and heart tissue of mice was measured via western blot analysis, as previously described (14). Lysis buffer [(for cells: Nonidet-P40 Cell Lysis Buffer (Thermo Fisher Scientific, Inc); for tissue: T-PER tissue protein extraction reagent (cat. no. 78510; Thermo Fisher Scientific, Inc.), $1 \mathrm{mM}$ phenylmethylsulfonylfluoride, phosphatase inhibitor cocktail (cat. no. 04906845001; Roche Diagnostics, Basel, Switzerland) and protease inhibitor cocktail (Pierce ${ }^{\mathrm{TM}}$ Protease Inhibitor Tablets, cat. no. 88265; Thermo Fisher Scientific, Inc.)] was used for lysis of cells and mammalian tissue and protein extraction. A bicinchoninic acid protein assay kit (Pierce; Thermo Fisher Scientific, Inc.) was used to determine the total protein concentration. Protein (30 mg) was subjected to $10-15 \%$ SDS-PAGE followed by electrophoresis and transfer onto nitrocellulose membranes (cat. no.03010040001, Roche Diagnostics). Membranes were blocked with Tris-buffered saline containing Tween-20 and 5\% bovine serum albumin for $2 \mathrm{~h}$ in room temperature. and then incubated with GAPDH (1:1,000 dilution), p-ERK1/2 (1:1,000 dilution), p-p38 (1:1,000 dilution), p-JNK (1:1,000 dilution), MMP-9 (1:1,000 dilution) and PCNA (1:1,000 dilution), Col1a1 (1:500 dilution) and $\alpha$-SMA (1:1,000 dilution) antibodies overnight at $4^{\circ} \mathrm{C}$. Membranes were then washed and incubated with HRP-labeled secondary antibodies (1:5,000 dilution) for $2 \mathrm{~h}$ at room temperature. Detection was performed using clarity western ECL substrate (Bio-Rad Laboratories, Hercules, CA, USA). Images were acquired and quantification analyses were performed using a ChemiDocMP system (Bio-Rad Laboratories).

Reverse transcription-quantitative polymerase chain reaction (RT-qPCR). The expression of Col1a1, Col3a1, transforming growth factor- $\beta$ (TGF- $\beta$ ), MMP-9 and $\alpha$-SMA was determined using RT-qPCR. Total RNA was extracted using TRIzol reagent (Invitrogen; Thermo Fisher Scientific, Inc.) according to the manufacturer's protocol. In total, $0.5 \mathrm{ng}$ RNA was used as a template for the synthesis of complementary (c)DNA using a first strand synthesis kit (cat. no. K1612; Invitrogen; Thermo Fisher Scientific, Inc.). qPCR analysis (ABI PRISM 7900 sequence detection system; Applied Biosystems; Thermo Fisher Scientific, Inc.) was performed on cDNA using PowerUp ${ }^{\mathrm{TM}} \mathrm{SYBR}^{\circledR}$ Green Master Mix (cat. no. A25742; Thermo Fisher Scientific, Inc.). The PCR conditions were $50^{\circ} \mathrm{C}$ for $2 \mathrm{~min}$ and $95^{\circ} \mathrm{C}$ for $10 \mathrm{~min}$, followed by 50 cycles of $95^{\circ} \mathrm{C}$ for $15 \mathrm{sec}$ and $60^{\circ} \mathrm{C}$ for $75 \mathrm{sec}$. The $2^{-\Delta \Delta \mathrm{Cq}}$ method was used to calculate the relative expression level of the transcripts (21). The mRNA expression of the target gene was normalized to endogenous GAPDH expression and represented as a fold-change relative to the control. Primers used for the amplification were as follows: Rat COL1 forward, 5'-CCC AAGGAAAAGAAGCACGTC-3' and reverse, 5'-AGGTCA GCTGGATAGCGACATC-3'; rat $\alpha$-SMA forward, 5'-GTC CCAGACATCAGGGAGTAA-3' and reverse, 5'-TCGGAT ACTTCAGCGTCAGGA-3'; rat MMP9 forward, 5'-CCTCTG CATGAAGACGACAT-3' and reverse, 5'-GAGGTGCAGTGG GACACATA-3'; rat GAPDH forward, 5'-GGCACAGTCAAG GCTGAGAATG-3' and reverse, 5'-GGCACAGTCAAGGCT GAGAATG-3'; rat ITGB3 forward, 5'-TGCAACAATGGT AACGGAAC-3' and reverse, 5'-CCTGCTGAGAGGGTCGAT AG-3'; mouse COL1 forward, 5'-GCTCCTCTTAGGGGCCAC T-3' and reverse, 5'-ATTGGGGACCCTTAGGCCAT-3'; mouse COL3 forward, 5'-CTGTAACATGGAAACTGGGGAAA-3' and reverse, 5'-CCATAGCTGAACTGAAAACCACC-3'; mouse TGF- $\beta$ forward, 5'-GGTCGCATCAAGGTGGTC TTT-3' and reverse, 5'-GTGGTGGTATTCCCCTTCTGG-3'; mouse GAPDH forward, 5'-AGGTCGGTGTGAACGGAT TTG-3' and reverse, 5'-TGTAGACCATGTAGTTGAGGTCA-3'.

For quantitative miRNA analysis, the Bulge-Loop ${ }^{\mathrm{TM}}$ miRNA qPCR Primer Set (Guangzhou RiboBio, Guangzhou, Guangdong, China) was used with the Takara SYBR Premix Ex Taq ${ }^{\mathrm{TM}}$ (Tli RNaseH Plus; Takara Bio Inc., Otsu, Japan) on an ABI-7900 Real-Time PCR Detection System (Applied Biosystems; Thermo Fisher Scientific, Inc.). A cDNA library was generated via RT using $20 \mu \mathrm{l}$ reaction system: $4 \mu \mathrm{l}$ $5 \mathrm{X}$ reaction buffer, $1 \mu \mathrm{l}$ RNA sample, $8 \mu \mathrm{l}$ RNase-free water, $2 \mu \mathrm{l}$ dNTPs, $2 \mu 1$ enzyme inhibitor, and $0.5 \mu 1 \mathrm{MMLV}$ reverse transcriptase (Takara Bio, Inc.) and $2.5 \mu \mathrm{l}$ specific RT primers of miRNA (Guangzhou RiboBio Co., Ltd.). The RT conditions were as follows: $42^{\circ} \mathrm{C}$ for $60 \mathrm{~min}$ followed by $95^{\circ} \mathrm{C}$ for $5 \mathrm{~min}$, then immediate cooling to $4^{\circ} \mathrm{C}$. Primers used for the amplification of miR-327 were as follows: mmu-miR-327 5'-ACUUGA GGGGCAUGAGGAU-3'; rno-miR-327, 5'-CCUUGAGGG GCAUGAGGGU-3'; U6 (cat. no. ssD0904071008, Guangzhou RiboBio Co., Ltd.) was used as an internal control for miRNA template normalization. The PCR conditions were as follows: 


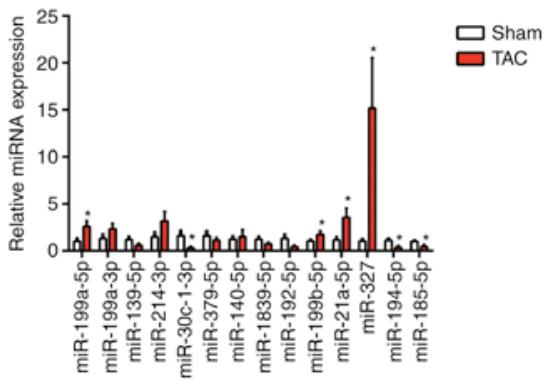

Figure 1. miR-327 is increased in cardiac hypertrophy. miR-327 was upregulated in the hearts of mice at 4 weeks following TAC surgery compared with that in sham control mice $(n=6) .{ }^{*} \mathrm{P}<0.05$ vs. Sham. miR, microRNA; TAC, transverse aortic constriction.

$95^{\circ} \mathrm{C}$ for $20 \mathrm{sec}$, followed by 40 cycles of $95^{\circ} \mathrm{C}$ for $10 \mathrm{sec}, 60^{\circ} \mathrm{C}$ for $20 \mathrm{sec}$ and $70^{\circ} \mathrm{C}$ for $10 \mathrm{sec}$. The $2^{-\Delta \Delta \mathrm{Cq}}$ method was used for analysis (21).
Statistical analysis. Values are expressed as the mean \pm standard error. Statistical analysis was performed using GraphPad Prism 5 (GraphPad Software, Inc., La Jolla, CA, USA). Statistical significance was determined using Student's t-test or one-way analysis of variance with Bonferroni's post-hoc test. $\mathrm{P}<0.05$ was considered to indicate a statistically significant difference.

\section{Results}

miR-327 expression is upregulated in fibrotic cardiac tissues. By using RT-qPCR analysis, a series of candidate miRNAs in the heart tissue of mice at 4 weeks were measured following TAC surgery. These candidate genes were the top 14 dysregulated miRNAs identified by miRNA arrays of post-MI ventricles with prominent fibrosis performed in a previous study (3). In the present study, miR-327 was identified as the highest upregulated miRNA in fibrotic heart tissue (Fig. 1). To confirm the cellular expression of miR-327, its expression was
A

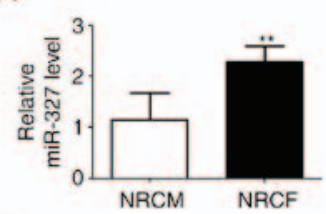

D

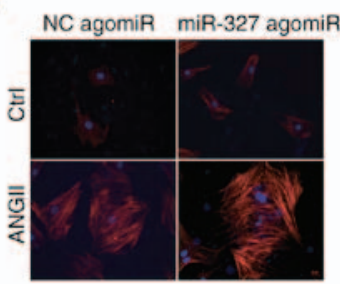

G
B

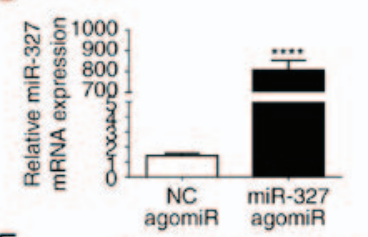

E

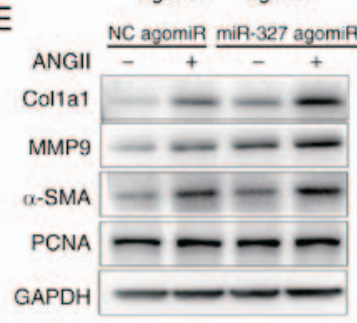

$\mathrm{H}$

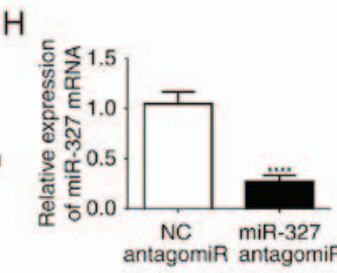

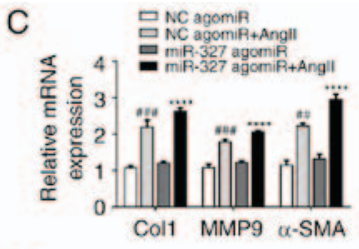

$\mathrm{F}$

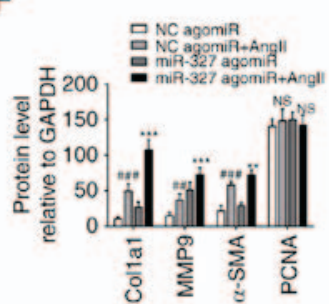

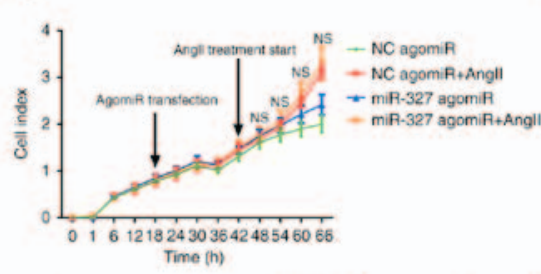
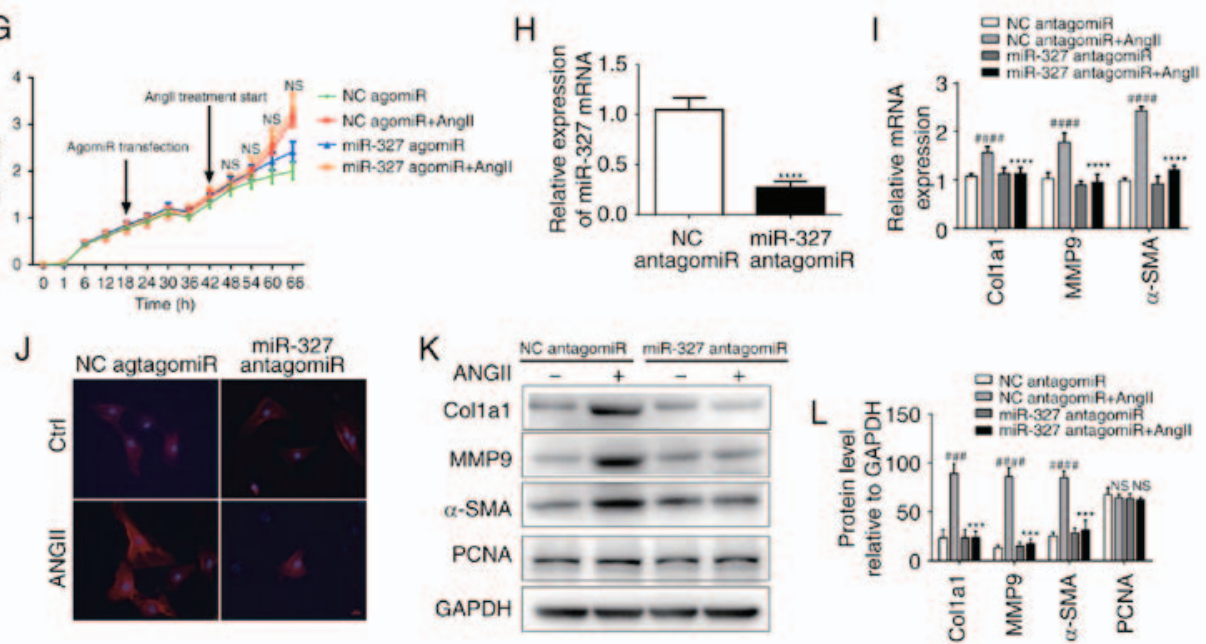

Figure 2. miR-327 promotes cardiac fibroblast differentiation into myofibroblasts in vitro. (A) miR-327 expression in NRCF and NRCM (n=6). (B) Overexpression of miR-327 with miR-327 agomiR $(n=6)$. (C) Effect of miR-327 agomiR treatment on markers of cardiac fibrosis during cardiac fibroblast differentiation as determined by RT-qPCR (n=6). (D) Fluorescence microscopy images demonstrating the effect of miR-327 overexpression on angiotensin expression during the differentiation of cardiac fibroblasts into myofibroblasts $(\mathrm{n}=3$; scale bar, $20 \mu \mathrm{m})$. (E and F) Western blot analysis indicated upregulation of fibrosis-associated markers in NRCF overexpressing miR-327 ( $\mathrm{n}=3)$. (G) Real-time cell proliferation assay using electric impedance as a measure of NRCF proliferation with miR-327 agomiR. ns, P>0.05, miR321-agomiR + AngII vs. NC-agomiR + AngII. (H) miR-327 antagomiR treatment decreased miR-327 in NRCFs (n=6). (I) Downregulation of fibrosis-associated gene expression in NRGF following inhibition of miR-327 (n=6). (J) Fluorescence microscopy images demonstrating inhibition of NRCF differentiation into myofibroblasts by inhibition of miR-327 (n=3; scale bar, $20 \mu \mathrm{m})$. (K and L) Downregulation of fibrosis-associated gene expression in NRCFs following inhibition of miR-327 ( $\mathrm{n}=3)$. ${ }^{\# \#} \mathrm{P}<0.01,{ }^{\# \# \#} \mathrm{P}<0.001,{ }^{\# \# \# \#} \mathrm{P}<0.0001$ vs. respective $\mathrm{NC}$ agomiR or $\mathrm{NC}$ antagomiR; ${ }^{* * *} \mathrm{P}<0.01,{ }^{* * * *} \mathrm{P}<0.001,{ }^{* * * * *} \mathrm{P}<0.0001$ vs. respective $\mathrm{NC}$ agomiR + AngII or NC antagomiR + Ang II. Ctrl, control; NC, negative control; ns, no significance; RT-qPCR, reverse transcription-quantitative polymerase chain reaction; Ang, angiotensin; col, collagen; $\alpha$-SMA, $\alpha$-smooth muscle actin; MMP, matrix metalloproteinase; miR, microRNA; PCNA, proliferating cell nuclear antigen; TGF, transforming growth factor; TAC, transverse aortic constriction; NRCF, neonatal cardiac fibroblasts; NRCM, neonatal cardiomyocytes. 
A

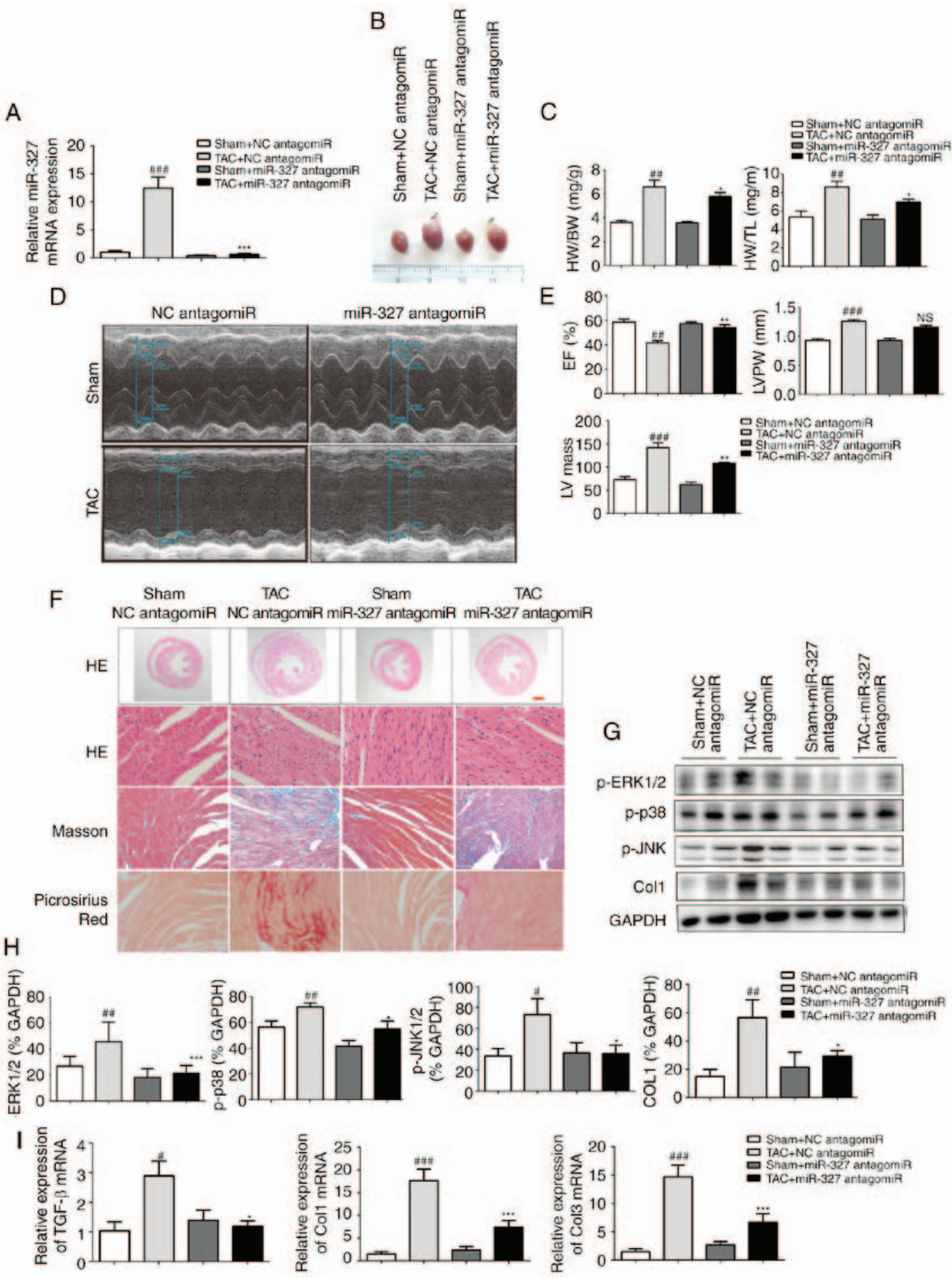

Figure 3. Cardiac hypertrophy is attenuated and ventricular function is preserved by miR-327 inhibition after TAC surgery. (A) Decreased miR-327 in hearts of mice treated with miR-327 antagomiR as determined by RT-qPCR $(\mathrm{n}=6)$. (B) Representative images of isolated mouse hearts (scale in mm). (C) miR-327 inhibition attenuated the HW/BW and HW/TL ratios $(\mathrm{n}=6)$. (D and E) Echocardiograms indicated that miR-327 inhibition preserved EF and attenuated LVPW and LV mass (n=6). (F) Histological examination indicated that miR-327 inhibition reduced cardiac hypertrophy and fibrosis $(\mathrm{n}=3$; scale bar, $500 \mu \mathrm{m}$ in the top panel and $50 \mu \mathrm{m}$ in the others). ( $\mathrm{G}$ and $\mathrm{H}$ ) Western blot analysis revealed that miR-327 inhibition prevented the phosphorylation of MAPK proteins (n=3). (I) miR-327 inhibition caused a downregulation of fibrosis-associated genes in mice ( $\mathrm{n}=6)$. ${ }^{\# /} \mathrm{P}<0.01,{ }^{\# \# t} \mathrm{P}<0.001$ vs. respective Sham $+\mathrm{NC}$ antagomiR; " $\mathrm{P}<0.05$, ${ }^{* *} \mathrm{P}<0.01,{ }^{* * * *} \mathrm{P}<0.001$ vs. respective TAC $+\mathrm{NC}$ antagomiR. ns, no significance; nc, negative control; col, collagen; ERK, extracellular signal-regulated kinase; JNK, c-Jun N-terminal kinase; miR, microRNA; TGF, transforming growth factor; TAC, transverse aortic constriction; RT-qPCR, reverse transcriptionquantitative polymerase chain reaction; EF, ejection fraction; HW, heart weight; BW, body weight; TL, tibia length; LVPW, left ventricular posterior wall; MAPK, mitogen-activated protein kinase; TGF, transforming growth factor.

examined in isolated neonatal rat cardiomyocytes and cardiac fibroblasts. The expression of miR-327 in cardiac fibroblasts was higher compared with that in the unstressed cardiomyocytes (Fig. 2A).
Differentiation of cardiac fibroblasts in vitro is attenuated by miR-327 inhibition. The development of myofibroblasts from fibroblasts is a critical step in the pathogenesis of cardiac fibrosis. In vitro experiments were used to improve the current 
$\begin{array}{cccc}\text { A miR-327 Mature sequence } & 3^{\prime} \quad \text {...UGGGAGUACGGGGAGUUCC...5' } \\ \text { 292-298 of ITGB3 } & 3^{\prime} \text { UTR } & 5^{\prime} & \text {...CUUAGCUUGAGGGGACUCAAGAU...3' } \\ \text { 586-593 of ITGB3 } & 3^{\prime} \text { UTR } & 5^{\prime} & \text {...GGGAGAACCCCGAGUCCUCAAGA...3' }\end{array}$

B

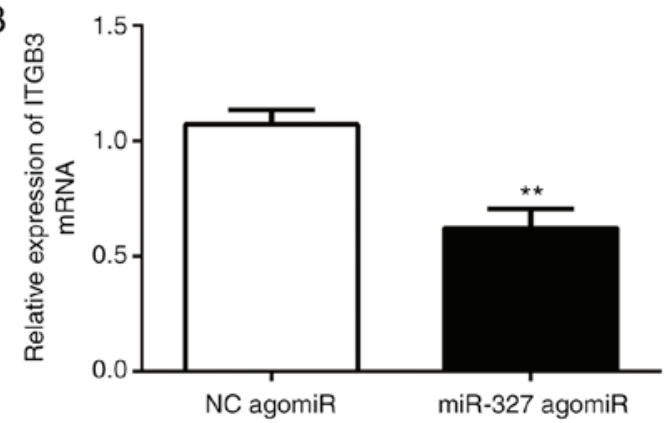

C

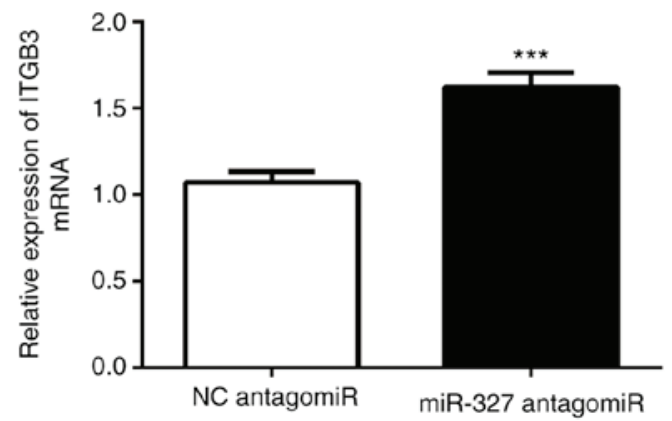

Figure 4. (A) Predicted miR-327 seed sequence matches the potential target gene mRNA. (B and C) miR-433 negatively regulated ITGB3 in neonatal cardiac fibroblasts $(\mathrm{n}=6) .{ }^{* *} \mathrm{P}<0.01,{ }^{* * *} \mathrm{P}<0.001$ vs. respective controls. ITGB3, integrin $\mathrm{B} 3$; miR, microRNA; UTR, untranslated region.

understanding of the role that miR-327 serves in regulating fibrosis. AgomiR-stimulated overexpression of miR-327 promoted angiotensin II-induced cardiac fibroblast differentiation, as indicated by the increase in $\alpha$-SMA expression and staining (Fig. 2B-D). The protein expression of MMP-9 and Colla1 was increased (Fig. 2E and F); however, cardiac fibroblast proliferation was not affected (Fig. 2G). By contrast, antagomiR-induced downregulation of miR-327 decreased cardiac fibroblast differentiation, although the proliferation remained unaffected (Fig. 2G-J). These results suggested that, in vitro, cardiac fibroblast differentiation into myofibroblasts was attenuated by miR-327 inhibition.

Cardiac hypertrophy and pressure overload-induced fibrosis are prevented by miR-327 inhibition. To investigate the effect of miR-327 on stressed hearts, miR-327 antagomiR was administered daily by tail-vein injection to mice for 3 days prior to TAC surgery. At 4 weeks following surgery, the loss of miR-327 in the heart was confirmed by RT-qPCR (Fig. 3A). Examination of the appearance of the hearts and the heart weight normalized to either body weight or tibia length demonstrated that miR-327 antagomiR administration prevented TAC-induced increases in LV volume and mass (Fig. 3B and C). Echocardiography revealed significantly improved $\mathrm{EF}, \mathrm{LV}$ mass and LV posterior wall thickness in miR-327 antagomiR-treated mice compared with those in the control mice (Fig. 3D and E).

miR-327 antagomiR-treated mice also possessed decreased cardiac chamber sizes and cardiomyocyte cross-sectional areas (Fig. 3F). The level of cardiac fibrosis was then assessed, given that this factor is critical in the progression of pathological cardiac remodeling. Masson trichrome and picrosirius red staining were used to visualize collagen deposition in LV sections. miR-327 antagomiR treatment attenuated TAC-induced fibrosis (Fig. 3F).

The mitogen-activated protein kinase (MAPK) cascade is a central signaling cascade known to promote the development of cardiac hypertrophy (22). To investigate the antihypertrophic effects of miR-327 antagomiR, the protein levels of activated MAPK members, including p-JNK, p-p38, and p-ERK1/2 were analyzed. Downregulation of miR-327 by antagomiR prevented phosphorylation of the MAPK proteins, which is typically caused by TAC (Fig. 3G and H). Finally, the mRNA levels of known pathological cardiac fibrosis markers, including TGF- $\beta$, Colla1 and Col3a1, were observed to be decreased in miR-327 antagomiR-treated mice (Fig. 3I).

miR-327 targets integrin (ITG)B3, contributing to its effect on cardiac fibrosis. A bioinformatics analysis with TargetScan (http://www.targetscan.org) suggested that ITGB3 is a direct target of miR-327. Cardiac fibroblasts were transfected with miR-327 agomiR, antagomiR or the scrambled control to determine whether miR-327 regulates ITGB3. As assessed via RT-qPCR, ITGB3 expression was increased by miR-327 antagomiR and decreased by miR-327 agomiR, suggesting the ability of miR-327 to regulate expression of endogenous ITGB3 in cardiac fibroblasts (Fig. 4B and C).

\section{Discussion}

Myocardial fibrosis is a frequent hallmark of cardiomyopathy (4). Although its original cause is known to be part of a physiological process, sustained stress induces pathological hypertrophy. In addition, interstitial fibrosis increases the stiffness of the myocardium. Subsequently, diastolic dysfunction-induced global cardiac remodeling leads to dilated cardiomyopathy and heart failure $(23,24)$. Despite the devastating nature of cardiac fibrosis, effective treatment strategies currently are not available. Thus, with regard to the treatment of cardiac dysfunction and heart failure, identification of therapeutic targets is of importance. Aberrant miRNA expression has been linked to cardiac fibrosis and heart failure $(9,25)$; Therefore, miRNAs have emerged as novel therapeutic targets for the treatment of fibrotic changes (6,26-28).

miRNAs are endogenous, non-coding small RNA molecules.miRs are recruited to the RNA-induced silencing complex and regulate target genes through diverse mechanisms (29). The importance of miRNAs in numerous cellular processes has been well documented. However, the significance of miRNAs in heart function, particularly with regard to cardiac fibrosis, has remained elusive. miR-122, miR-145, miR-134, miR-133a and miR-370 were reported to be associated with coronary artery disease, even following adjustment for other 
cardiovascular risk factors (30-33). Dysregulated miRNAs, particularly miR-21 and miR-29b, were previously indicated to contribute to cardiac fibrosis $(34,35)$. Increased expression of miR-21 in fibroblasts promotes their proliferation, suggesting the important role of miR-21 in cardiac remodeling (36,37). By miRNA arrays, it was previously demonstrated that high levels miR-327 are associated with hypertrophic cardiac tissues (3). In the present study investigated whether miR-327 serves a role in the development of pathological cardiac hypertrophy and fibrosis. Using in vivo and in vitro models, it was demonstrated that miR-327 antagomiR inhibited cardiac hypertrophy and fibrosis. Cardiac fibrosis occurs in numerous types of heart disease, including diabetic cardiomyopathy, MI, dilated cardiomyopathy, aortic stenosis and hypertrophic cardiomyopathy $(38,39)$. In cardiac fibrosis, cardiac fibroblasts proliferate and transform into myofibroblasts (40). The present study demonstrated that cardiac fibroblast differentiation, which was enhanced by miR-327 overexpression, was attenuated by miR-327 inhibition. In addition, miR-327 levels were enhanced in cardiac fibroblasts compared with those in cardiomyocytes. Esposito et al (41) indicated that TAC-induced pressure overload is associated with the activation of MAPKs (ERK1/2, p38 MAPK and JNK) in mice. Consistent with numerous animal studies, clinical studies reported that in failing human hypertrophic hearts, ERK1/2, JNK and p38 MAPK were significantly activated (42). In line with these results, MAPK activation has been demonstrated to be a key element directly enhancing cardiac remodeling $(43,44)$. Through a bioinformatics analysis performed in the present study, ITGB3 was identified as a target gene for miR-327 in cardiac fibroblasts. ITGB3, which is an ITGB-chain subunit encoded by a serotonin-associated gene on chromosome 17, was reported to be linked with the risk of cancer, including colorectal cancer and acute myeloid leukemia $(45,46)$. The ITGB3 family includes the subtypes $\alpha$ IIb $\beta 3$ and $\alpha v \beta 3$. The ITGB3 gene encodes the integrin $\beta 3$ subunit and has been consistently identified as a quantitative locus for regulating serum levels of 5-hydroxytryptamin levels (47). In the present study, ITGB3 was inhibited by miR-327 antagomiR, suggesting that ITGB3 may be a target gene of miR-327 inducing differentiation of cardiac fibroblasts. However, the direct association between ITGB3 and MAPK and their functional roles in the regulation of cardiac fibrosis require further confirmation.

In summary, the present study suggested that cardiac fibrosis may increase miR-327 expression and that miR-327 antagomiR improves LV mass and attenuates cardiac fibrosis during cardiac hypertrophy. The protective effect of miR-327 inhibition against cardiac fibrosis was mediated through inhibition of TAC-induced increases in ERK1/2, p38 and JNK phosphorylation. ITGB3 may be a target gene of miR-327 that induces differentiation of cardiac fibroblasts. Thus, a previously unknown role for miR-327 in cardiac hypertrophy and fibrosis was identified in the present study, but this requires to be confirmed in future studies.

\section{Acknowledgements}

The present study was supported by grants from the National Natural Science Foundation of China (grant nos. 81627802 and 81570247), the Natural Science Foundation of Jiangsu
Province for Youth (grant no. BK20141024), the Six Talent Peaks project in Jiangsu Province (grant no. 2015-WSN-29) and the Priority Academic Program Development of Jiangsu Higher Education Institutions.

\section{Competing interests}

The authors declare that they have no competing interests.

\section{References}

1. Huang S, Zou X, Zhu JN, Fu YH, Lin QX, Liang YY, Deng CY, Kuang SJ, Zhang MZ, Liao YL, et al: Attenuation of microrna-16 derepresses the cyclins $\mathrm{d} 1, \mathrm{~d} 2$ and e1 to provoke cardiomyocyte hypertrophy. J Cell Mol Med 19: 608-619, 2015.

2. Chien KR, Shimizu M, Hoshijima M, Minamisawa S and Grace AA: Toward molecular strategies for heart disease-past, present, future. Jpn Circ J 61: 91-118, 1997.

3. Tao L, Bei Y, Chen P, Lei Z, Fu S, Zhang H, Xu J, Che L, Chen X, Sluijter JP, et al: Crucial role of mir-433 in regulating cardiac fibrosis. Theranostics 6: 2068-2083, 2016.

4. Thannickal VJ, Zhou Y, Gaggar A and Duncan SR: Fibrosis: Ultimate and proximate causes. J Clin Invest 124: 4673-4677, 2014.

5. Thum $\mathrm{T}$ and Lorenzen JM: Cardiac fibrosis revisited by microRNA therapeutics. Circulation 126: 800-802, 2012.

6. Olson EN: Micrornas as therapeutic targets and biomarkers of cardiovascular disease. Sci Transl Med 6: 239ps3, 2014.

7. Yang X, Wu D, Du H, Nie F, Pang X and Xu Y: MicroRNA-135a is involved in podocyte injury in a transient receptor potential channel 1-dependent manner. Int J Mol Med 40: 1511-1519, 2017.

8. Zhou B, Li H and Shi J: miR-27 inhibits the NF- $\kappa$ B signaling pathway by targetingleptin in osteoarthritic chondrocytes. Int J Mol Med 40: 523-530, 2017.

9. Thum T: Noncoding RNAs and myocardial fibrosis. Nat Rev Cardiol 11: 655-663, 2014.

10. Tao H, Yang JJ and Shi KH: Non-coding RNAs as direct and indirect modulators of epigenetic mechanism regulation of cardiac fibrosis. Expert Opin Ther Targets 19: 707-716, 2015.

11. Kumarswamy R and Thum T: Non-coding RNAs in cardiac remodeling and heart failure. Circ Res 113: 676-689, 2013.

12. Espinoza-Lewis RA and Wang DZ: MicroRNAs in heart development. Curr Top Dev Biol 100: 279-317, 2012.

13. Small EM and Olson EN: Pervasive roles of microRNAs in cardiovascular biology. Nature 469: 336-342, 2011.

14. Shi J, Wang L, Lu Y, Ji Y, Wang Y, Dong K, Kong X and Sun W: Protective effects of seabuckthorn pulp and seed oils against radiation-induced acute intestinal injury. J Radiat Res 58: 24-32, 2017.

15. Bourajjaj M, Armand AS, da Costa Martins PA, Weijts B, van der Nagel R, Heeneman S, Wehrens XH and De Windt LJ: Nfatc2 is a necessary mediator of calcineurin-dependent cardiac hypertrophy and heart failure. J Biol Chem 283: 22295-22303, 2008.

16. Lu Y, Zhu X, Li J, Fang R, Wang Z, Zhang J, Li K, Li X, Bai H, Yang $\mathrm{Q}$, et al: Glycine prevents pressure overload induced cardiac hypertrophy mediated by glycine receptor. Biochem Pharmacol 123: 40-51, 2017.

17. Zaid TM, Yeung TL, Thompson MS, Leung CS, Harding T, Co NN, Schmandt RS, Kwan SY, Rodriguez-Aguay C, LopezBerestein G, et al: Identification of FGFR4 as a potential therapeutic target for advanced-stage, high-grade serous ovarian cancer. Clin Cancer Res 19: 809-820, 2013.

18. Cheng Y, Zhu Y, Zhang J, Duan X and Zhang Y: Large accumulation of collagen and increased activation of mast cells in hearts of mice with hyperlipidemia. Arq Bras Cardiol 109: 404-409, 2017 (In English, Portuguese).

19. Irelan JT, Wu MJ, Morgan J, Ke N, Xi B, Wang X, Xu X and Abassi YA: Rapid and quantitative assessment of cell quality, identity, and functionality for cell-based assays using real-time cellular analysis. J Biomol Screen 16: 313-322, 2011.

20. Xing D and Orsulic S: A genetically defined mouse ovarian carcinoma model for the molecular characterization of pathwaytargeted therapy and tumor resistance. Proc Natl Acad Sci USA 102: 6936-6941, 2005.

21. Livak KJ and Schmittgen TD: Analysis of relative gene expression data using real-time quantitative PCR and the $2^{-\Delta \Delta C_{\mathrm{T}}}$ method. Methods 25: 402-408, 2001. 
22. Heineke J and Molkentin JD: Regulation of cardiac hypertrophy by intracellular signalling pathways. Nat Rev Mol Cell Biol 7: 589-600, 2006.

23. Maillet M, van Berlo JH and Molkentin JD: Molecular basis of physiological heart growth: Fundamental concepts and new players. Nat Rev Mol Cell Biol 14: 38-48, 2013.

24. Dirkx E, Gladka MM, Philippen LE, Armand AS, Kinet V, Leptidis S, El Azzouzi H, Salic K, Bourajjaj M, da Silva GJ, et al: Nfat and mir-25 cooperate to reactivate the transcription factor hand2 in heart failure. Nat Cell Biol 15: 1282-1293, 2013.

25. Melman YF, Shah R and Das S: Micrornas in heart failure: Is the picture becoming less mirky? Circ Heart Fail 7: 203-214, 2014.

26. Roncarati R, Viviani Anselmi C, Losi MA, Papa L, Cavarretta E, Da Costa Martins P, Contaldi C, Saccani Jotti G, Franzone A, Galastri L, et al: Circulating miR-29a, among other up-regulated microRNAs, is the only biomarker for both hypertrophy and fibrosis in patients with hypertrophic cardiomyopathy. J Am Coll Cardiol 63: 920-927, 2014.

27. Wang J, Liew OW, Richards AM and Chen YT: Overview of MicroRNAs in cardiac hypertrophy, fibrosis, and apoptosis. Int J Mol Sci 17: pii: E749, 2016.

28. Wu C, Dong S and Li Y: Effects of miRNA-455 on cardiac hypertrophy induced by pressure overload. Int J Mol Med 35: 893-900, 2015.

29. Ling H, Fabbri M and Calin GA: Micrornas and other non-coding RNAs as targets for anticancer drug development. Nat Rev Drug Discov 12: 847-865, 2013.

30. Gacoń J, Kabłak-Ziembicka A, Stępień E, Enguita FJ, Karch I, Derlaga B, Żmudka K and Przewłocki T: Decision-making microRNAs (miR-124, -133a/b, -34a and -134) in patients with occluded target vessel in acute coronary syndrome. Kardiol Pol 74: 280-288, 2016.

31. Gao H, Guddeti RR, Matsuzawa Y, Liu LP, Su LX, Guo D, Nie SP, Du J and Zhang M: Plasma levels of microRNA-145 are associated with severity of coronary artery disease. PLoS One 10: e0123477, 2015.

32. Gao W, He HW, Wang ZM, Zhao H, Lian XQ, Wang YS, Zhu J, Yan JJ, Zhang DG, Yang ZJ and Wang LS: Plasma levels of lipometabolism-related miR-122 and mir-370 are increased in patients with hyperlipidemia and associated with coronary artery disease. Lipids Health Dis 11: 55, 2012.

33. Wang F, Long G, Zhao C, Li H, Chaugai S, Wang Y, Chen C and Wang DW: Plasma microRNA-133a is a new marker for both acute myocardial infarction and underlying coronary artery stenosis. J Transl Med 11: 222, 2013.

34. Thum T, Gross C, Fiedler J, Fischer T, Kissler S, Bussen M, Galuppo P, Just S, Rottbauer W, Frantz S, et al: MicroRNA-21 contributes to myocardial disease by stimulating map kinase signalling in fibroblasts. Nature 456: 980-984, 2008.

35. Wang J, Huang W, Xu R, Nie Y, Cao X, Meng J, Xu X, Hu S and Zheng Z: MicroRNA-24 regulates cardiac fibrosis after myocardial infarction. J Cell Mol Med 16: 2150-2160, 2012.
36. Ye Y, Perez-Polo JR, Qian J and Birnbaum Y: The role of microRNA in modulating myocardial ischemia-reperfusion injury. Physiol Genomics 43: 534-542, 2011.

37. Dong S, Cheng Y, Yang J, Li J, Liu X, Wang X, Wang D, Krall TJ, Delphin ES and Zhang C: MicroRNA expression signature and the role of microRNA-21 in the early phase of acute myocardial infarction. J Biol Chem 284: 29514-29525, 2009.

38. Kong P, Christia P and Frangogiannis NG: The pathogenesis of cardiac fibrosis. Cell Mol Life Sci 71: 549-574, 2014.

39. Gyöngyösi M, Winkler J, Ramos I, Do QT, Firat H, McDonald K, González A, Thum T, Diez J, Jaisser F, et al: Myocardial fibrosis: Biomedical research from bench to bedside. Eur J Heart Fail 19: 177-191, 2017.

40. Weber KT, Sun Y, Bhattacharya SK, Ahokas RA and Gerling IC: Myofibroblast-mediated mechanisms of pathological remodelling of the heart. Nat Rev Cardiol 10: 15-26, 2013.

41. Esposito G, Perrino C, Schiattarella GG, Belardo L, di Pietro E, Franzone A, Capretti G, Gargiulo G, Pironti G, Cannavo A, et al: Induction of mitogen-activated protein kinases is proportional to the amount of pressure overload. Hypertension 55: 137-143, 2010.

42. Haq S, Choukroun G, Lim H, Tymitz KM, del Monte F, Gwathmey J, Grazette L, Michael A, Hajjar R, Force T and Molkentin JD: Differential activation of signal transduction pathways in human hearts with hypertrophy versus advanced heart failure. Circulation 103: 670-677, 2001.

43. Peterson KL: Pressure overload hypertrophy and congestive heart failure. Where is the 'Achilles' heel'? J Am Coll Cardiol 39: 672-675, 2002.

44. Rose BA, Force T and Wang Y: Mitogen-activated protein kinase signaling in the heart: Angels versus demons in a heart-breaking tale. Physiol Rev 90: 1507-1546, 2010.

45. Lei Y, Huang K, Gao C, Lau QC, Pan H, Xie K, Li J, Liu R, Zhang T, Xie N, et al: Proteomics identification of ITGB3 as a key regulator in reactive oxygen species-induced migration and invasion of colorectal cancer cells. Mol Cell Proteomics 10: M110 005397, 2011.

46. Miller PG, Al-Shahrour F, Hartwell KA, Chu LP, Järås M, Puram RV, Puissant A, Callahan KP, Ashton J, McConkey ME, et al: In vivo RNAi screening identifies a leukemia-specific dependence on integrin beta 3 signaling. Cancer Cell 24: 45-58, 2013.

47. Mazalouskas M, Jessen T, Varney S, Sutcliffe JS, VeenstraVanderWeele J, Cook EH Jr and Carneiro AM: Integrin $\beta 3$ haploinsufficiency modulates serotonin transport and antidepressant-sensitive behavior in mice. Neuropsychopharmacology 40 : 2015-2024, 2015.

This work is licensed under a Creative Commons Attribution-NonCommercial-NoDerivatives 4.0 International (CC BY-NC-ND 4.0) License. 